\title{
Radiocesium sorption behavior on illite, kaolinite, and their mixtures
}

\author{
N.K. Ishikawa, S. Uchida and K. Tagami \\ National Institute of Radiological Sciences, Anagawa 4-9-1, Inage-ku, Chiba, Japan
}

\begin{abstract}
Clay minerals are one of the most important components in soil for radiocesium sorption. There are many types of clay minerals in soil, and their capacities for cesium (Cs) sorption differ. However, the effects of differing clay mineral amounts and compositions on Cs sorption behavior have not been clarified yet. In this study, therefore, we studied Cs sorption on illite, kaolinite, and illite-kaolinite mixtures, carrying out batch sorption test and sequential extractions to investigate Cs sorption mechanisms. The amount of sorbed Cs in the mixtures did not depend on the illite content, but the amount of exchangeable Cs decreased as the illite content increased. Conversely, the amount of fixed Cs in the mixtures increased with increasing illite content. These results suggest that the distribution of Cs sorption between ion exchange sites and fixed sites may be dependent on the illite/kaolinite ratio. In addition, we can estimate the amount of sorbed Cs in an illite-kaolinite mixture from the amounts of sorbed Cs in illite and in kaolinite, and the abundance ratio of each clay mineral in the clay mixture. This is basic knowledge needed for long-term assessment of Cs mobility in soil.
\end{abstract}

\section{INTRODUCTION}

Cesium-135 $\left({ }^{135} \mathrm{Cs}\right.$, half-life: $\left.2 \times 10^{6} \mathrm{y}\right)$ and ${ }^{137} \mathrm{Cs}$ (half-life: $\left.30 \mathrm{y}\right)$ are produced by nuclear fission in high yield. Therefore, knowledge of the mobility of radiocesium in the natural environment is needed to assess the impact of its release from waste disposal sites [1]. Since one of the most important pathways of radiocesium in the environment to humans is via food consumption, knowledge of the behavior of Cs in agricultural fields is needed. To understand plant uptake of Cs from soil, knowledge of not only the amount of sorbed Cs in the soil but also the sorption mechanism is needed.

It is well known that Cs can sorb strongly onto soil, especially clay minerals [2, 3]. Clay minerals have two types of sorption sites for Cs: the Regular Exchange Complex (REC) sites which are planar and/or interlayer, and the Frayed Edge Sites (FESs) which are located at the edge and/or interlayer of the micaceous clay minerals such as illite [4]. FESs selectively sorb $\mathrm{Cs}^{+}, \mathrm{K}^{+}$, and $\mathrm{NH}_{4}^{+}$, and they cannot subsequently release these ions into the soil solution. This phenomenon is called fixation. The complex sorption behavior of Cs on clay minerals makes Cs sorption in soil difficult to estimate. Indeed, the soilsoil solution distribution coefficient, which is defined as the ratio of the adsorbed Cs concentration to the Cs concentration in the soil solution, does not correlate with the clay content in Japanese agricultural soils [5]. Therefore, it is necessary to investigate Cs sorption on individual clay mineral types, and on mixtures of clay minerals.

The objective of this study was to quantitatively estimate Cs sorption in mixtures of clay minerals. We used two different clay minerals, illite and kaolinite, that are typically present in Japanese agricultural fields; we previously found that among 30 Japanese paddy field soil samples, illite and kaolinite were included in 30 and 14 samples, respectively (unpublished data). It is well known that illite has both REC sites and FESs, whereas kaolinite has REC sites but not FESs. Clarifying Cs sorption and fixation in the illite-kaolinite system can therefore be basic knowledge needed for long-term assessment of Cs mobility in soil. 


\section{EXPERIMENTAL}

\subsection{Materials}

Illite and kaolinite were purchased from Hayashi Shouten Co. Ltd. (Osaka, Japan) and Iwamoto Kousan Co. Ltd. (Tokyo, Japan), respectively. Illite is a $2: 1$ clay mineral and kaolinite is a $1: 1$ clay mineral. Table 1 summarizes the chemical compositions and cation exchange capacities (CEC). Prior to the experiments, the clay minerals were washed with deionized water to remove excess salt. About $25 \mathrm{~g}$ of each clay mineral was mixed with about $200 \mathrm{~mL}$ of deionized water in a $250-\mathrm{mL}$ plastic bottle. The suspension was shaken at 120 revolutions per minute (rpm) for 1 day at $23^{\circ} \mathrm{C}$ using an end-over-end shaker. The suspension was then centrifuged at $10000 \mathrm{rpm}$ (HITACHI, himac CR 21E) for $10 \mathrm{~min}$ and the supernatant was discarded. This washing process was repeated until the electrical conductivity (EC) of the supernatant become constant. Finally, the residue was air-dried at room temperature.

Table 1. Chemical compositions and CECs of the clay minerals used in this study.

\begin{tabular}{|l|c|c|c|c|c|c|c|c|c|c|}
\hline & \multicolumn{7}{|c|}{ Element (wt/\%) } & \multirow{2}{*}{ CEC (meq/100 g) } \\
\cline { 2 - 10 } & $\mathrm{Al}$ & $\mathrm{Si}$ & $\mathrm{S}$ & $\mathrm{K}$ & $\mathrm{Ca}$ & $\mathrm{Ti}$ & $\mathrm{Fe}$ & $\mathrm{Rb}$ & $\mathrm{Sr}$ & 35 \\
\hline Illite & 19.3 & 51.4 & 0.1 & 20.4 & 2.4 & 1.5 & 4.2 & 0.1 & 0.2 & 4.4 \\
\hline Kaolinite & 32.1 & 60.8 & 0.3 & 0.6 & 0.1 & 2.8 & 2.6 & ${ }^{\dagger}$ n.d. & 0.3 & 0.3 \\
\hline
\end{tabular}

†n.d.: not detected.

\subsection{Batch sorption test}

We carried out batch sorption tests using ${ }^{137} \mathrm{Cs}$ as a tracer. Illite and kaolinite mixtures containing from 0 to $100 \%$ illite were prepared, shaken (as described below), and then $100 \mathrm{mg}$ of each mixture and 10 $\mathrm{mL}$ of deionized water were transferred to a $50-\mathrm{mL}$ centrifuge tube. The suspension was first shaken at $120 \mathrm{rpm}$ for 1 day at $23^{\circ} \mathrm{C}$ using an end-over-end shaker, and then $0.1 \mathrm{~mL}$ of ${ }^{137} \mathrm{Cs}$ solution, with $10 \mathrm{mg} / \mathrm{L}$ stable Cs as a carrier, was added to each tube. The samples were then shaken for 7 more days. Preliminary trials (data not shown) revealed that this was a sufficiently long period to reach sorption equilibrium in the sample suspension. After the shaking, the phases were separated by centrifuging at 10 $000 \mathrm{rpm}$ for $20 \mathrm{~min}$, and the supernatant was filtered through a $0.20-\mu \mathrm{m}$ membrane filter (ADVANTEC, DISMIC-25AS). The radioactivity of ${ }^{137} \mathrm{Cs}$ in the filtrate was measured with an NaI scintillation counter (Aloka, ARC-380). The $\mathrm{pH}$ and EC of each filtrate were measured with a pH meter (Horiba, B-212) and an EC meter (Horiba, B-173), respectively. During the experiment, pH ranged from 6.1 to 6.4, and EC ranged from 1.4 to $2.2 \mathrm{mS} / \mathrm{m}$. The value $Q$ is given by:

$$
Q=\frac{Q_{s}}{W_{s}}
$$

where $Q_{s}$ is the amount of Cs in solid phase and $W_{s}$ is the weight of solid sample.

\subsection{Sequential extraction method}

To determine the exchangeable (weakly sorbed, strongly sorbed), and fixed Cs fractions, a sequential extraction method was used [6]. The solid phase after the batch sorption test was added to $10 \mathrm{~mL}$ of $0.05 \mathrm{M}$ calcium chloride $\left(\mathrm{CaCl}_{2}\right)$ and shaken for $24 \mathrm{~h}$ at $23{ }^{\circ} \mathrm{C}$. The suspension was then centrifuged at $10000 \mathrm{rpm}$ for $20 \mathrm{~min}$, and the supernatant, which contained the weakly sorbed fraction, was filtered through a $0.20-\mu \mathrm{m}$ membrane filter. The strongly sorbed fraction in the residual solid phase of the sample was extracted for $24 \mathrm{~h}$ at $23^{\circ} \mathrm{C}$ with $30 \mathrm{~mL}$ of $0.16 \mathrm{M}$ sodium pyrophosphate $\left(\mathrm{Na}_{4} \mathrm{P}_{2} \mathrm{O}_{7}\right)$. The suspension from this extraction was processed in the same way as for the $\mathrm{CaCl}_{2}$ extraction. Radioactivity of ${ }^{137} \mathrm{Cs}$ in the filtrates was measured with the NaI scintillation counter. Cs in the residue after pyrophosphate 
extraction was assumed to be the fraction fixed in the clay minerals. The three fractions are related as follows:

$$
Q=q_{\mathrm{ex}}+q_{\mathrm{fix}}
$$

$Q$; total amount of sorbed Cs

$q_{\text {ex }}$ : total amount of exchangeable $\mathrm{Cs}\left(=q_{\text {ex.w }}+q_{\text {ex.s }}\right)$

$q_{\text {ex.w }}$ : amount of weakly sorbed $\mathrm{Cs}$

$q_{\text {ex.s }}:$ amount of strongly sorbed Cs

$q_{\text {fix }}:$ amount of fixed Cs.

\section{RESULTS AND DISCUSSION}

\subsection{Sorbed Cs in clay mixtures}

Figure 1 shows the variation in the amount of sorbed Cs $(Q)$ in the mixtures in relation to the illite content. Almost $100 \%$ of the Cs was sorbed in all mixtures; thus $Q$ did not differ in relation to the illite content. However, $q_{\text {ex }}$ decreased from 9.8 to $5.8 \mathrm{mg} / \mathrm{kg}$ with increasing illite content, whereas $q_{\text {fix }}$ increased from 0.1 to $4.2 \mathrm{mg} / \mathrm{kg}$ with increasing illite content. These results suggest that although the total amount of sorbed Cs was similar in all mixtures regardless of the illite/kaolinite ratio, the distribution of $\mathrm{Cs}$ between the exchangeable and fixed fractions varied with the illite/kaolinite ratio. Since almost all of the sorbed $\mathrm{Cs}$ in pure kaolinite (0\% sample in Fig. 1) was extracted by $\mathrm{CaCl}_{2}$ and $\mathrm{Na}_{4} \mathrm{P}_{2} \mathrm{O}_{7}$, it was confirmed that $0.16 \mathrm{M} \mathrm{Na}_{4} \mathrm{P}_{2} \mathrm{O}_{7}$ can extract Cs sorbed on REC sites in clay minerals. In pure illite $(100 \%$ sample in Fig. 1$), q_{\mathrm{ex}}$ and $q_{\mathrm{fix}}$ were 5.8 and $4.2 \mathrm{mg} / \mathrm{kg}$, respectively. Thus, the amount of Cs in REC sites was about 1.4 times that in FESs in the illite used in this study. These results indicated that the amount of Cs sorption by REC sites is not negligible compared with that by FESs in clay mixtures, in contrast to the results obtained for soil and sediment samples by Wauters et al. [7], because $58 \%$ of the sorbed $\mathrm{Cs}$ in pure illite was in the exchangeable fraction.

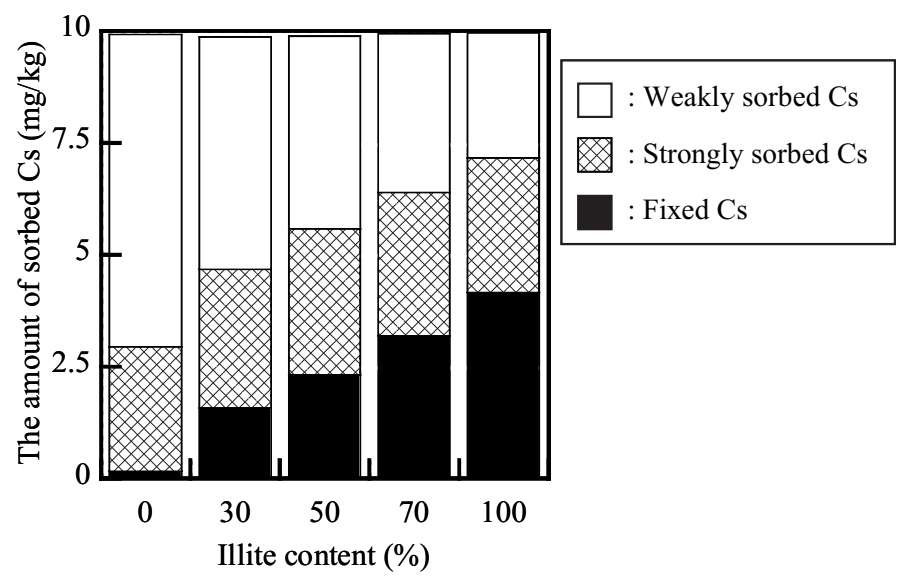

Figure 1. The amount of sorbed Cs in clay mixtures in relation to illite content.

\subsection{Estimation of the amount of sorbed Cs in clay mixtures}

Clay minerals cannot block each other's sorption sites in clay mixtures because almost all surfaces of clay minerals are negatively charged. Therefore, if the relative abundance of each clay mineral in a 
clay mixture is known, the total amount of sorbed Cs in the clay mixture can be estimated from the amounts of Cs sorbed on illite and kaolinite. If the amount of Cs sorbed on $100 \%$ kaolinite is $Q_{\mathrm{K} 100, \mathrm{I} 0}$ and the amount of Cs sorbed on $100 \%$ illite is $Q_{\mathrm{K} 0, \mathrm{I} 100}$, then the amount of sorbed Cs in a mixture of $x \%$ kaolinite and $y \%$ illite (where $x+y=100 \%$ ), $Q_{\mathrm{Kx}, \mathrm{Iy}}$, can be estimated by using the following equation.

$$
Q_{\mathrm{Kx}, \mathrm{Iy}}=\left(x \times Q_{\mathrm{K} 100, \mathrm{I} 0}+y \times Q_{\mathrm{K} 0, \mathrm{I} 100}\right) / 100
$$

However, as described above, $Q$ did not depend on the illite/kaolinite ratio under the experimental conditions of this study. Thus, we could not estimate $Q$ values because $Q$ values were within the error range.

In contrast, $q_{\mathrm{ex}}$ decreased, and $q_{\mathrm{fix}}$ increased, linearly with increasing illite content (Fig. 2). Similar to $Q$ with equation (3), $q_{\mathrm{ex}}$ and $q_{\mathrm{fix}}$ in a mixture of illite and kaolinite can be estimated by using the following equations.

$$
\begin{aligned}
& q_{\mathrm{ex}(\mathrm{Kx}, \mathrm{Iy})}=\left(x \times q_{\mathrm{ex}(\mathrm{K} 100, \mathrm{I} 0)}+y \times q_{\mathrm{ex}(\mathrm{K} 0, \mathrm{I} 100)}\right) / 100 \\
& q_{\mathrm{fix}(\mathrm{Kx}, \mathrm{Iy})}=\left(x \times q_{\mathrm{fix}(\mathrm{K} 100, \mathrm{I} 0)}+y \times q_{\mathrm{fix}(\mathrm{K} 0, \mathrm{I} 100)}\right) / 100
\end{aligned}
$$

Figure 3 shows the relationships between values of $q_{\mathrm{ex}}$ and $q_{\mathrm{fix}}$ estimated with equations (4) and (5) and those obtained by sequential extraction. The correlation coefficient $(r)$ of both relationships is 1.00 , indicating that $q_{\mathrm{ex}}$ and $q_{\mathrm{fix}}$ values can be estimated for the illite-kaolinite mixtures if the amounts of exchangeable and fixed $\mathrm{Cs}$ in pure illite and pure kaolinite and the ratio of illite to kaolinite in the mixture are known. Though this method can be used to estimate $Q$ values in a simple illite-kaolinite mixture, it is difficult to estimate $Q$ values in more complicated systems such as soil. One reason is that soil contains many different clay minerals, each with a different affinity for Cs. Another is that soil organic matter can potentially sorb onto clay minerals, thus blocking sorption sites and inhibiting Cs sorption by clays [8]. Further study is needed to enable the estimation of $Q$ values in more complicated systems such as mixtures of clay and soil organic matter.

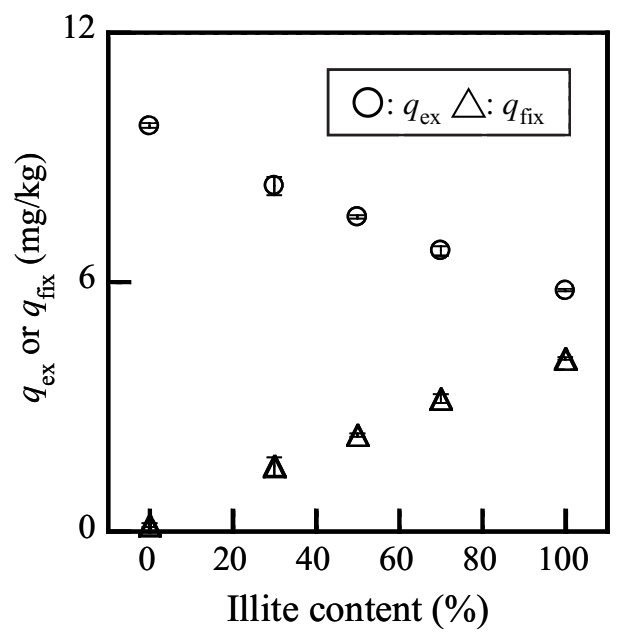

Figure 2. Relationship between $q_{\mathrm{ex}}$ or $q_{\mathrm{fix}}$ and illite content.

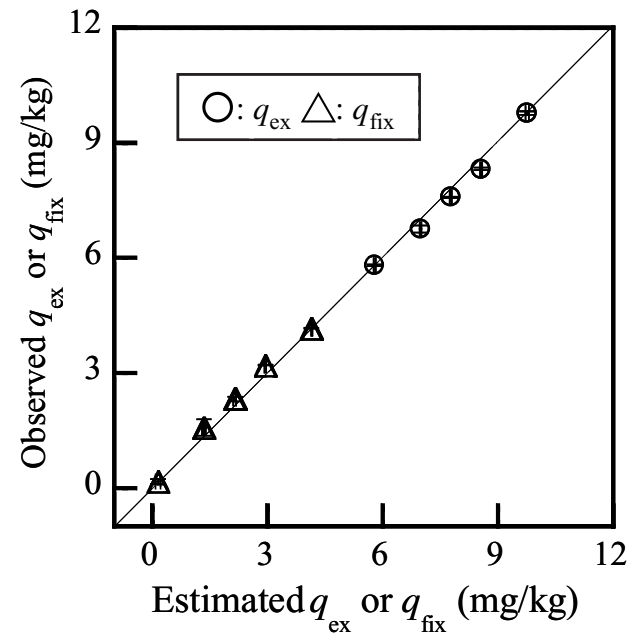

Figure 3. Comparison of observed and estimated $q_{\mathrm{ex}}$ or $q_{\mathrm{fix}}$ values. 


\subsection{Distribution of sorbed Cs between REC sites and FESs in the clay mixtures}

As reported by Wauters et al. [7], FESs have a much stronger affinity for Cs than REC sites. However, in the present results, considerable amounts of Cs were sorbed on REC sites. It is probable that the distribution of Cs sorption between RECs and FESs changes as the ratio of the number of REC sites to the number of FESs in the clay mixture changes.

The $q_{\mathrm{ex}} / q_{\text {fix }}$ ratio decreased with increasing illite content (Fig. 4). Thus, the distribution of Cs sorption between REC sites and FESs was dependent on the illite/kaolinite ratio, presumably because the area ratio of REC sites to FESs depends on the illite/kaolinite ratio. As a result, the relative abundance of each clay mineral is important for estimating Cs mobility in mixtures of clay minerals. This knowledge is important for long-term assessment of Cs mobility in soil.

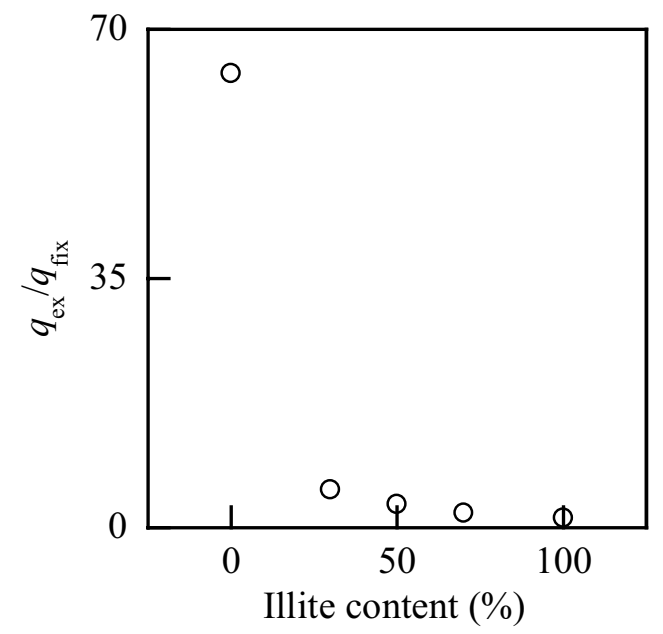

Figure 4. Relationship between illite content and $q_{\mathrm{ex}} / q_{\mathrm{fix}}$.

\section{Acknowledgments}

This work was partly supported by the Agency for Natural Resources and Energy, the Ministry of Economy, Trade and Industry (METI), Japan. The authors thank Dr. N. Satta from Iwate University for measuring the cation exchangeable capacity of the illite and kaolinite.

\section{References}

[1] Japan Nuclear Cycle Development Institute, The second progress report on research and development for the HLW disposal in Japan, vol. 3 (JNC, Tokai, 1999) pp. 327-329.

[2] Tamura T., Nuclear Safety 5 (1964) 262-268.

[3] Cornell R.M., J. Radioanal. Nucl. Chem. 171 (1993) 483-500.

[4] Delvaux B., Kruyts M., Maers E. and Smolders E., Trace elements in the rhizosphere (CRC Press, Bocan Raton, 2001) pp. 61-91.

[5] Ishikawa N.K., Uchida S. and Tagami K., in Proceedings of the Waste Management Conference, Phoenix, 2008, in press

[6] Ishikawa N.K., Uchida S. and Tagami K., J. Radioanal. Nucl. Chem. (2008) in press

[7] Wauters J., Elsen A., Cremers A., Konoplev A.V., Bulgakov A.A. and Comans R.N.J., Appl. Geochem. 11 (1996) 589-594.

[8] Staunton S., Dumat C. and Zsolnay A., J. Environ. Radioact. 58 (2002) 163-173. 
